\title{
Exposição a agrotóxicos ou práticas agroecológicas: ideação suicida entre camponeses do semiárido no Brasil
}

\author{
Exposure to pesticides or agroecological practices: suicidal ideation \\ among peasant farmers in Brazil's semi-arid region
}

Carla Wernecke Padovani Gonzaga (https://orcid.org/0000-0003-0263-6021) ${ }^{1}$

Marcelo Perim Baldo (https://orcid.org/0000-0002-7673-3580) ${ }^{1}$

Antônio Prates Caldeira (https://orcid.org/0000-0002-9990-9083) ${ }^{1}$
${ }^{1}$ Universidade Estadual de Montes Claros. Av. Prof. Rui Braga s/n, Vila Mauriceia. 39401-089 Montes Claros MG Brasil.

carlawpado@gmail.com

\begin{abstract}
Health risks faced by peasant farmers may vary depending on the type of agriculture they practice. This study examined the association between suicide ideation and exposure to pesticides by comparing two groups of peasant farmers of both sexes living in the semi-arid region of the north of Minas Gerais, Brazil: exposed to pesticides and adopting agroecological practices without the use of pesticides. Group participants were selected using convenience sampling and data was collected using a previously validated questionnaire administered through face-to-face interviews. Bivariate analysis was performed, followed by logistic regression. A total of 547 peasant farmers were interviewed (311 in the group exposed to pesticides and 236 in the group adopting agroecological practices). Respondents from the group exposed to pesticides were more likely to report suicidal ideation ( $O R=2.30$; 95\%CI 1.164.56), harmful alcohol consumption $(O R=2.30$; 95\% CI 1.18-4.48), and lifetime acute pesticide poisoning ( $O R=8.58 ; 95 \% C I$ 2.98-24.72). The findings suggest that agricultural practices that lead to chronic pesticide exposure are associated with a greater likelihood of suicide ideation, regardless of previous episodes of acute pesticide poisoning or harmful alcohol consumption.
\end{abstract}

Key words Suicide, Environmental exposure, Acute poisoning, Sustainable agriculture, Rural populations
Resumo Diferentes práticas agrícolas estão associadas a diferentes riscos para a saúde de camponeses. Objetivou-se analisar a associação entre ideação suicida e exposição a agrotóxicos, comparando camponeses vivendo sob exposição ambiental a agrotóxicos em relação a camponeses envolvidos com práticas agroecológicas, sem o uso de agrotóxicos, no semiárido norte mineiro. As famílias avaliadas foram amostradas, por conveniência, a partir da prática agrícola a qual estavam vinculadas, e os dados coletados por meio de entrevistas com instrumento previamente validado. Foram realizadas análises bivariadas seguidas de regressão logística. Foram entrevistados 547 camponeses, de ambos os sexos, sendo 311 sob exposição a agrotóxicos. Os camponeses expostos a agrotóxicos, em relação a camponeses envolvidos com práticas agroecológicas, tiveram maiores chances para ideação suicida $(\mathrm{OR}=2,30$; IC95\% 1,164,56), consumo problemático de álcool $(\mathrm{OR}=2,30$; IC95\% 1,18-4,48) e episódios prévios de intoxicação aguda por agrotóxicos (OR=8,58; IC95\% 2,98-24,72).Conclui-se que as práticas agrícolas que implicam em exposição crônica a agrotóxicos se associam a maiores chances de ideação suicida, independentemente de episódios prévios de intoxicação aguda por agrotóxicos ou do uso problemático do álcool.

Palavras-chave Suicídio, Exposição ambiental, Intoxicação aguda, Agricultura Sustentável, População rural 


\section{Introdução}

Os efeitos dos agrotóxicos na saúde humana têm sido uma preocupação crescente na medida em que o consumo desses produtos cresce no mundo e, mais enfaticamente, no Brasil, que se consagrou como o maior mercado consumidor de agrotóxicos do mundo, desde $2008^{1,2}$. Entre 2007 e 2017, foram registrados no Brasil cerca de 40 mil casos de intoxicação por agrotóxicos, com média de 148 mortes ao ano ${ }^{3}$. Há crescentes evidências que associam exposição a agrotóxicos com agravos à saúde, como câncer ${ }^{4,5}$, desregulação endócrina ${ }^{6,7}$, desordens neurológicas ${ }^{8,9}$, alterações comportamentais e neuropsiquiátricas ${ }^{10-12}$ e distúrbios do neurodesenvolvimento ${ }^{13}$.

O uso de agrotóxicos remete à complexidade das relações de vida e trabalho no campo, o que incita a se fazer certas distinções entre modelos de desenvolvimento agrícola e os seus respectivos impactos na saúde dos camponeses. A agricultura industrial e a agricultura agroecológica apresentam-se como modelos opostos de organização da agricultura, especialmente no que se refere às suas relações com os ecossistemas ${ }^{14}$. A agricultura industrial pressupõe a especialização do agricultor no cultivo de um único produto, configurando-se em monoculturas e divisão de tarefas, com vistas aos ganhos de produtividade. A agricultura agroecológica pressupõe a biodiversidade e estimula a interação entre plantas e outros organismos para gerar fertilidade nas áreas de cultivo, preservando os ecossistemas, garantindo os meios de subsistência aos camponeses e fixando maior número de pessoas no campo ${ }^{15}$. A agroecologia prescinde do uso de agrotóxicos e busca fortalecer a agricultura familiar em diversificadas experiências e iniciativas nos territórios ${ }^{16}$.

Estudos apontam que o trabalho na agricultura é um fator de risco para suicídio ${ }^{17,18}$. Todavia, existe um risco maior de morte por suicídio entre trabalhadores rurais que residem em áreas de maior exposição a agrotóxicos ${ }^{19}$. Precedendo o suicídio ou a tentativa de suicídio, habitualmente, existe a ideação suicida, que é a presença de pensamentos passivos ou ativos sobre o fim prematuro da vida. Trata-se de um tema pouco explorado na população de áreas rurais no Brasil, contando com uma prevalência de 5\% $\%^{20,21}$. Ainda que a ideação suicida seja um fator de risco para o suicídio, bem como para morbidades em geral e todas as causas de mortalidade ${ }^{22}$, um complexo de muitos outros fatores contribui para esses desfechos. Estudos têm apontado aassociação entre intoxicação aguda por agrotóxicos e distúrbios psiquiátricos ${ }^{23-25}$. Entretanto, existe ainda uma lacuna na literatura quanto à associação entre distúrbios psiquiátricos e a exposição crônica, isto é, a exposição prolongada e cumulativa a baixas doses de agrotóxicos ${ }^{26}$. Assim, este estudo teve o propósito de analisar a frequência de ideação suicida entre camponeses vivendo sob exposição ambiental a agrotóxicos, em relação a camponeses envolvidos com práticas agroecológicas.

\section{Métodos}

Trata-se de um estudo transversal e analítico, realizado entre janeiro e março de 2019, desenhado para comparar duas populações rurais do semiárido no extremo norte de Minas Gerais, envolvidas em modelos agrícolas diferentes. Foram selecionadas, de forma independente, as seguintes populações rurais: 1) camponeses envolvidos com práticas agrícolas tradicionais, segundo o modelo da agricultura agroecológica; 2) camponeses vivendo sob exposição ambiental a agrotóxicos, segundo o modelo de agricultura industrial.

Ao tomar para o estudo dois grupos populacionais do semiárido, com semelhantes origens históricas, procurou-se assegurar maior similaridade quanto às características etnográficas, sociodemográficas e culturais entre eles, excetuando-se o fato de estarem, ambos, envolvidos com práticas agrícolas diferentes nos últimos 40 anos.

\section{Características das áreas de estudo}

O vale do rio Gorutuba no semiárido mineiro abriga um projeto federal de agricultura irrigada, nos moldes da agricultura industrial, privilegiando as monoculturas e o uso intensivo de agrotóxicos. No território do Projeto de agricultura irrigada no vale do Gorutuba, implantado após a construção da Barragem Bico da Pedra em 1979, residem camponeses que se empregam como trabalhadores rurais nas lavouras de bananas e outras frutas, ou, mais raramente, são proprietários de pequenos lotes agrícolas. As residências são construídas dentro dos lotes agrícolas, contíguas aos campos de cultivo, o que torna aqueles que aí residem vulneráveis à contaminação pelas pulverizações aéreas, praticadas rotineiramente na região ${ }^{27}$. As águas do rio Gorutuba servem tanto à agricultura quanto ao uso doméstico (banho, lavagem de roupas e utensílios de cozinha) e fluem pelo território através de canaletas abertas que recebem a deriva das pulverizações de agrotóxi- 
cos, configurando um cenário de ampla exposição, incluindo a contaminação do solo, ar, água, alimentos, animais e seres humanos.

Para além do perímetro de agricultura irrigada, o semiárido abriga diversas comunidades rurais tradicionais que manejam a agricultura familiar, em estreita relação de preservação dos recursos naturais e práticas extrativistas sustentáveis, prescindindo do uso de agrotóxicos.

A ideia subjacente ao desenho do estudo é de que camponeses residentes dentro do perímetro de agricultura irrigada, imersos no modelo de agricultura industrial, independentemente de trabalharem diretamente nas lavouras, experimentam baixas-doses de exposição prolongada e cumulativa a agrotóxicos, por meio da absorção respiratória, transdérmica e oral ${ }^{28,29}$.

\section{Populações do estudo}

No território irrigado habitavam 1.264 pessoas adultas, distribuídas em 755 famílias, convivendo com 4.242 hectares plantados de bananas e outras frutas, em janeiro de 2019. Foram incluídos no estudo camponeses, homens e mulheres, com idade de 18 anos ou mais, residentes no território há mais de dois anos, independentemente de exercerem atividades diretas na agricultura. As 10 microáreas da Estratégia de Saúde da Família que compunham o perímetro irrigado foram incluídas no estudo, sendo os camponeses entrevistados por Agentes Comunitários de Saúde locais que se dispuseram a colaborar com a pesquisa. Não foram considerados, para o presente estudo, os camponeses que, embora trabalhassem dentro do perímetro irrigado, não residiam naquele território.

Para o grupo de práticas agroecológicas, foram selecionados camponeses oriundos de quatro comunidades rurais, distribuídas em três outros municípios do semiárido: Comunidade do Touro, em Serranópolis; Comunidade Córrego Verde, em Grão Mogol; Comunidade Fazenda Velha e Comunidade Nossa Senhora das Oliveiras (antigo assentamento Tapera), ambos no município de Riacho dos Machados. Tais camponeses eram reconhecidos pelas associações locais de moradores, em suas comunidades de origem, por suas práticas agroecológicas com o manejo sustentável dos recursos naturais e sem o uso de agrotóxicos.

Camponeses, com idade igual ou acima de 18 anos, foram convidados a participar do estudo durante reuniões públicas nas comunidades, ocasião em que o projeto de pesquisa foi apresentado a todos. Foram excluídos do grupo agroe- cológico: 1) camponeses empregados na empresa mineradora instalada no território, uma vez que as atividades mineradoras podem acarretar agravos à saúde que levariam a vieses; 2) camponeses que participaram da colheita sazonal de café ou corte de cana-de-açúcar, nos últimos dois anos, considerando que foram potencialmente expostos a agrotóxicos.

O cadastro populacional das 1.264 pessoas adultas moradoras no território do perímetro irrigado não foi disponibilizado para fins deste estudo. A amostragem se deu por conveniência, sem intencionalidade, em ambos os grupos, considerando a dificuldade de estabelecer um plano amostral, pela distribuição aleatória dos domicílios em área rural. Todavia, buscou-se representatividade de todas as microáreas da Estratégia de Saúde da Família no perímetro irrigado, por meio da colaboração dos Agentes Comunitários de Saúde. Para o grupo agroecológico, não foram encontrados registros oficiais de quantas pessoas viviam em cada uma das comunidades tradicionais, sendo o número de moradores presumido em cerca de 600 pessoas, a partir de informações das próprias comunidades. Nessas comunidades foram alocados todos os indivíduos que se enquadravam nos critérios de inclusão alcançáveis a partir das indicações das lideranças locais.

\section{Instrumento de coleta de dados}

O instrumento de coleta de dados foi construído a partir do objetivo do trabalho, com base em instrumentos previamente validados. As variáveis estudadas foram divididas em socioeconômicas/demográficas e aquelas relacionadas às condições de saúde. No primeiro grupo de variáveis foram incluídas: sexo (masculino $\mathrm{x}$ feminino), idade (de 18 a 39 anos $x \geq 40$ anos), cor da pele (brancos $\mathrm{x}$ pardos/pretos/indígenas/ amarelos), estado conjugal (com companheiro $\mathrm{x}$ sem companheiro), escolaridade (alfabetizado $\mathrm{x}$ analfabeto). No grupo das variáveis relacionadas à saúde, foram incluídas, além da ideação suicida, outras variáveis que potencialmente podem interferir com tal evento: tabagismo atual, uso problemático de álcool e intoxicação prévia por agrotóxico (todas dicotômicas: sim x não).

A ideação suicida foi aferida a partir de uma pergunta contida no Self Reporting Questionnaire-20 $0^{30}$ : "Alguma vez você pensou em acabar com a sua vida nos últimos 30 dias?", com opções de resposta: sim e não. Trata-se de uma pergunta contextualizada no questionário proposto pela Organização Mundial de Saúde e validado 
no Brasil, cuja finalidade é acessar transtornos mentais comuns, não psicóticos, na Atenção Primária. A forma assertiva e contextualizada para abordagem da ideação suicida na Atenção Primária tem sido preconizada para avaliação do risco de suicídio.

A intoxicação aguda por agrotóxicos é um indicador de altos níveis de exposição a agrotóxicos e a presença de intoxicação aguda prévia por agrotóxicos, em algum momento da vida, foi aferida por meio de uma pergunta do Protocolo de Avaliação das Intoxicações Crônicas por Agrotóxicos do Estado do Paraná ${ }^{30}$, com opções de resposta: sim e não.

O uso problemático de álcool foi aferido a partir da escala Alcohol Use Disorders Identification Test (AUDIT), para identificar o consumo problemático de álcool no âmbito da Atenção Primária, em que o escore maior ou igual a oito pontos, para ambos os sexos, era preditor de problemas médicos e sociais relacionados ao álcool ${ }^{31}$. A presença de tabagismo atual foi aferida por meio do Protocolo de Avaliação das Intoxicações Crônicas por Agrotóxicos do Estado do Paraná ${ }^{30}$.

\section{Coleta de dados}

O trabalho de campo ocorreu entre os meses de janeiro e março de 2019, por meio de entrevistas face-a-face, conduzidas por Agentes Comunitários de Saúde e moradores locais, devidamente treinados e supervisionados para coletar as informações junto às suas comunidades de origem, após obtenção escrita do Consentimento Livre e Esclarecido para participação na pesquisa. Os entrevistadores foram orientados a realizarem as entrevistas nos domicílios dos moradores, onde mais de um adulto poderia ser entrevistado, em horários que fossem mais adequados aos respondentes. Cerca de 5\% dos questionários foram, aleatoriamente, selecionados para controle da qualidade das informações, não sendo identificados equívocos ou inconsistências.

\section{Análise dos dados}

A análise descritiva das características das populações estudadas foi realizada pela distribuição de frequências e análise bivariada das variáveis independentes categóricas, comparando-se os grupos (Camponeses de comunidades tradicionais com práticas agroecológicas x Camponeses potencialmente expostos a agrotóxicos na agricultura industrial) por meio do teste Qui-quadrado de Pearson.
Todas as variáveis estudadas entraram inicialmente no modelo de regressão logística binária e aquelas com níveis de significância maior que 5\% foram excluídas do modelo final, exceto as variáveis sexo e idade que, independentemente dos níveis de significância, foram mantidas no modelo final como fatores de ajuste.

Os dados coletados foram analisados através do programa estatístico Statistical Package for Social Sciences (SPSS), versão 18.0.

\section{Aspectos éticos}

O projeto foi submetido e aprovado pelo Comitê de Ética em Pesquisa da Universidade Estadual de Montes Claros (UNIMONTES). Todos os respondentes consentiram com a participação no estudo, por meio de assinatura ou registro da digital em Termo de Consentimento Livre e Esclarecido. Ao final da coleta de dados, foram realizadas palestras educativas nas comunidades sobre os riscos à saúde decorrentes do uso e exposição a agrotóxicos.

\section{Resultados}

Foram entrevistadas 547 pessoas, sendo 311 no grupo de camponeses vivendo sob exposição a agrotóxicos no território do Projeto de agricultura irrigada, segundo o modelo de agricultura industrial, e 236 pessoas no grupo de camponeses envolvidos com práticas agroecológicas nas comunidades tradicionais do semiárido. As características socioeconômicas e demográficas dos grupos estão apresentadas na Tabela 1. Os grupos de camponeses mostraram-se semelhantes quanto a distribuição por sexo e idade. Em ambos os grupos, cerca de $40 \%$ dos indivíduos vivem em lares com renda familiar mensal abaixo de um salário mínimo. Mais de $85 \%$ dos indivíduos em cada grupo declararam-se pardos, pretos, amarelos ou indígenas. As proporções de analfabetismo e famílias monoparentais (pessoas vivendo sem um companheiro) foram maiores entre camponeses das comunidades tradicionais do semiárido com práticas agroecológicas em relação aos camponeses expostos a agrotóxicos no território do Projeto de agricultura irrigada (Tabela 1).

Na Tabela 2 estão descritas condições associadas à saúde dos camponeses. Os grupos se mostraram estatisticamente diferentes em relação a todas as características avaliadas: uso problemático de álcool, tabagismo atual, intoxicação prévia por agrotóxicos e ideação suicida 
Tabela 1. Caracterização socioeconômica e demográfica de camponeses e associação com práticas agrícolas (práticas agroecológicas x exposição a agrotóxicos) no semiárido do norte de Minas Gerais; 2019 (n=547) (Análises bivariadas).

\begin{tabular}{|c|c|c|c|c|c|c|}
\hline Variáveis & $\mathbf{n}(\%)$ & $\begin{array}{c}\text { Agroecologia } \\
\text { n }(\%)\end{array}$ & $\begin{array}{c}\text { Agrotóxicos } \\
\text { n }(\%)\end{array}$ & OR bruta & IC95\% & $\mathbf{p}^{*}$ \\
\hline \multicolumn{7}{|l|}{ Sexo } \\
\hline Masculino & $232(42,4)$ & $92(39,0)$ & $140(45,0)$ & 1 & - & \\
\hline Feminino & $315(57,6)$ & $144(61,0)$ & $171(55,0)$ & 0,78 & $0,55-1,10$ & 0,16 \\
\hline \multicolumn{7}{|l|}{ Grupo etário } \\
\hline 18 a 39 anos & $251(47,0)$ & $111(47,0)$ & $140(45,0)$ & 1 & - & \\
\hline$\geq 40$ anos & $296(53,0)$ & $125(53,0)$ & $171(55,0)$ & 1,09 & $0,77-1,52$ & 0,64 \\
\hline \multicolumn{7}{|l|}{ Cor da pele } \\
\hline Branco & $48(8,08)$ & $26(11,0)$ & $22(7,1)$ & 1 & - & \\
\hline Não branco & $499(91,2)$ & $207(87,7)$ & $288(92,6)$ & 1,63 & $0,90-2,95$ & 0,11 \\
\hline \multicolumn{7}{|l|}{ Estado conjugal } \\
\hline Com companheiro & $362(66,2)$ & $143(60,6)$ & $219(70,4)$ & 1 & - & \\
\hline Sem companheiro & $185(33,8)$ & $93(39,4)$ & $92(29,6)$ & 0,65 & $0,45-0,92$ & 0,02 \\
\hline \multicolumn{7}{|l|}{ Escolaridade $^{\star}$} \\
\hline Alfabetizado & $480(89,2)$ & $201(85,5)$ & $279(92,1)$ & 1 & - & \\
\hline Analfabeto & $58(10,8)$ & $34(14,5)$ & $24(7,9)$ & 0,51 & $0,29-0,88$ & 0,02 \\
\hline \multicolumn{7}{|l|}{ Renda familiar } \\
\hline 1 ou mais salários & $314(57,4)$ & $137(58,1)$ & $177(56,9)$ & 1 & - & \\
\hline$<1$ salário mínimo & $233(42,6)$ & $99(41,9)$ & $134(43,1)$ & 1,05 & $0,74-1,48$ & 0,79 \\
\hline
\end{tabular}

Fonte: Elaborada pelos autores.

Tabela 2. Caracterização de condições relacionadas à saúde de camponeses e associação com práticas agrícolas (práticas agroecológicas x exposição a agrotóxicos) no semiárido do norte de Minas Gerais; 2019 (n=547) (Análises bivariadas).

\begin{tabular}{|c|c|c|c|c|c|c|}
\hline Variáveis & $\mathbf{n}(\%)$ & $\begin{array}{c}\text { Agroecologia } \\
\mathbf{n}(\%)\end{array}$ & $\begin{array}{c}\text { Agrotóxicos } \\
\text { n (\%) }\end{array}$ & OR bruta & IC95\% & $\mathbf{p}^{*}$ \\
\hline \multicolumn{7}{|c|}{ Uso problemático de álcool } \\
\hline Não & $491(89,8)$ & $221(93,6)$ & $270(86,8)$ & 1 & - & - \\
\hline $\operatorname{Sim}$ & $56(10,2)$ & $15(6,4)$ & $41(13,2)$ & 2,24 & $1,21-4,15$ & 0,01 \\
\hline \multicolumn{7}{|l|}{ Tabagismo atual ${ }^{\star}$} \\
\hline Não & $467(85,8)$ & $209(89,7)$ & $258(83,0)$ & 1 & - & - \\
\hline $\operatorname{Sim}$ & $77(14,2)$ & $24(10,3)$ & $53(17,0)$ & 1,79 & $1,07-3,00$ & 0,03 \\
\hline \multicolumn{7}{|c|}{ Intoxicação aguda por agrotóxicos } \\
\hline Não & $499(91,2)$ & $232(98,3)$ & $267(85,9)$ & 1 & - & \\
\hline $\operatorname{Sim}$ & $48(8,8)$ & $4(1,7)$ & $44(14,1)$ & 9,56 & $3,39-27,00$ & 0,00 \\
\hline \multicolumn{7}{|c|}{ Ideação suicida nos últimos 30 dias $^{*}$} \\
\hline Não & $490(90,1)$ & $221(94,4)$ & $269(86,8)$ & 1 & - & - \\
\hline $\operatorname{Sim}$ & $54(9,9)$ & $13(5,6)$ & $41(13,2)$ & 2,59 & $1,35-4,96$ & 0,00 \\
\hline
\end{tabular}

*Variação no $\mathrm{n}=547$, devido à perda de informação.

Fonte: Elaborada pelos autores.

nos últimos 30 dias. As maiores proporções de aspectos negativos relacionados à saúde foram registradas entre os camponeses residentes no território do Projeto de agricultura industrial ir- rigada, quando comparados àqueles oriundos de comunidades tradicionais do semiárido. Entre os camponeses das comunidades tradicionais, 1,7\% relatou ter sofrido episódio de intoxicação aguda 
por agrotóxico em algum momento da vida, anteriormente à adoção das práticas agroecológicas.

Após análise múltipla, a chance de ideação suicida, nos últimos 30 dias, ajustada por sexo, idade, estado conjugal, escolaridade, uso problemático de álcool e intoxicação aguda prévia por agrotóxicos foi maior ( $\mathrm{OR}=2,30$; IC95\% 1,16$4,56)$ entre os camponeses expostos a agrotóxicos no território de agricultura industrial irrigada, quando comparada à chance de ideação suicida entre camponeses de comunidades tradicionais com práticas agroecológicas no semiárido.

Outras variáveis que se mantiveram estatisticamente associadas ao contexto das práticas agrícolas foram: estado conjugal, escolaridade e intoxicação aguda prévia por agrotóxicos. Sexo e idade permaneceram como fatores de ajuste na análise de regressão logística, mesmo não alcançando níveis de significância estatística (Tabela 3).

\section{Discussão}

Este estudo identificou maior frequência de ideação suicida entre camponeses vivendo sob exposição ambiental a agrotóxicos em relação a camponeses envolvidos com práticas agroecológicas. Essa associação, que pressupõe uma exposição crônica a agrotóxicos, ainda não havia sido registrada no país, embora trabalhadores rurais vivendo em áreas com altas taxas de uso de agrotóxicos, na região Serrana do Rio de Janeiro, tenham apresentado maiores riscos de mortalidade e hospitalizações por tentativas de suicídio, sendo que o risco de morte aumentou com o grau de exposição a agrotóxicos ${ }^{19}$. No Mato Grosso do Sul, Brasil, foram encontradas maiores prevalências de tentativas de suicídio e suicídio em áreas de alto consumo de agrotóxicos, em relação a áreas de baixo consumo ${ }^{32}$. Na Espanha, o estudo ecológico com 17.429 pessoas, encontrou maiores taxas de suicídio e tentativas de suicídio em áreas agrícolas de uso intensivo de agrotóxicos ${ }^{9}$.

$\mathrm{Na}$ China, um estudo transversal de base populacional, com 10.000 pessoas de comunidades rurais, mostrou associação entre exposição a agrotóxicos e ideação suicida nos últimos dois $\operatorname{anos}^{21}$. Nos Estados Unidos, a exposição ocupacional a agrotóxicos esteve associada ao aumento do risco de depressão ${ }^{33}$. No Canadá, observou-se aumento nas taxas de mortalidade por suicídio em aplicadores de herbicidas e inseticidas, em relação a agricultores em geral $^{34}$.

A literatura associa ideação suicida a episódio de intoxicação aguda por agrotóxicos. No estudo
Tabela 3. Características associadas às práticas agrícolas (práticas agroecológicas $\mathrm{x}$ exposição a agrotóxicos) no semiárido do norte de Minas Gerais; 2019 ( $n=547)$ (Análise de regressão logística).

\begin{tabular}{|c|c|c|c|}
\hline \multirow[t]{2}{*}{ Variáveis } & \multicolumn{3}{|c|}{$\begin{array}{c}\text { Camponeses expostos a } \\
\text { agrotóxicos/Camponeses } \\
\text { agroecológicos }\end{array}$} \\
\hline & $\begin{array}{c}\mathrm{OR} \\
\text { ajustada }\end{array}$ & IC95\% & $\mathbf{p}$ \\
\hline \multicolumn{4}{|l|}{ Sexo } \\
\hline Masculino & 1 & - & - \\
\hline Feminino & 0,95 & $0,62-1,32$ & 0,61 \\
\hline \multicolumn{4}{|l|}{ Grupo etário } \\
\hline 18 a 39 anos & 1 & - & - \\
\hline$\geq 40$ anos & 1,01 & $0,69-1,48$ & 0,95 \\
\hline \multicolumn{4}{|l|}{ Estado conjugal } \\
\hline Com companheiro & 1 & - & - \\
\hline Sem companheiro & 0,65 & $0,44-0,95$ & 0,03 \\
\hline \multicolumn{4}{|l|}{ Escolaridade $^{\star}$} \\
\hline Alfabetizado & & - & - \\
\hline Analfabeto & 0,43 & $0,23-0,81$ & 0,01 \\
\hline \multicolumn{4}{|l|}{$\begin{array}{l}\text { Uso problemático de } \\
\text { álcool }\end{array}$} \\
\hline Não & & - & - \\
\hline Sim & 2,30 & $1,18-4,48$ & 0,01 \\
\hline \multicolumn{4}{|l|}{$\begin{array}{l}\text { Intoxicação aguda por } \\
\text { agrotóxicos }\end{array}$} \\
\hline Não & & - & - \\
\hline $\operatorname{Sim}$ & 8,58 & $2,98-24,72$ & 0,00 \\
\hline \multicolumn{4}{|l|}{$\begin{array}{l}\text { Ideação suicida nos } \\
\text { últimos } 30 \text { dias }^{\star}\end{array}$} \\
\hline Não & & - & - \\
\hline Sim & 2,30 & $1,16-4,56$ & 0,02 \\
\hline
\end{tabular}

*Variação no $n=547$, devido à perda de informação.

Fonte: Elaborada pelos autores.

transversal com trabalhadores de plantações de banana na Costa Rica, indivíduos que sofreram intoxicações agudas por agrotóxicos organofosforados tiveram maiores chances para pensamentos suicidas, no último mês, comparados aos trabalhadores sem história de intoxicação aguda por estes agrotóxicos ${ }^{25}$. Na Coreia do Sul, o risco para ideação suicida, no último ano, aumentou com a severidade da intoxicação aguda e história de hospitalização por intoxicação aguda por agrotóxicos ${ }^{20}$. O estudo de Faria et al. ${ }^{17}$ evidenciou associação entre exposição a agrotóxicos e taxas padronizadas de suicídio nas regiões brasileiras, com maior magnitude da associação entre intoxicação aguda por agrotóxicos e suicídio.

Embora as avaliações realizadas neste estudo não tenham incluído dosagens de metabólitos 
de agrotóxicos, a contaminação ambiental do ar, água, solo e alimentos, bem como a proximidade das residências em relação às lavouras são suficientes para presumir tal exposição $0^{1,3,5,9,27-29,35-38}$ Os camponeses vivendo no território do Projeto de agricultura industrial irrigada no vale do Gorutuba foram considerados ambientalmente expostos a agrotóxicos, independentemente de trabalharem diretamente com o preparo ou aplicação destes produtos. Essa questão foi estudada em uma coorte de neonatos nos Países Baixos, onde se investigou a influência de fatores ambientais no desenvolvimento de doenças respiratórias, e observou-se que a proximidade entre as residências e os campos de cultivo tratados com agrotóxicos foi um marcador de exposição ambiental a agrotóxicos ${ }^{28}$.

No Brasil, um estudo ecológico evidenciou que o aumento do consumo de agrotóxicos se correlaciona positivamente com os coeficientes médios dos seguintes indicadores de saúde: intoxicação aguda por agrotóxicos, incidência de malformação fetal e câncer infantojuvenil ${ }^{35}$. No Ceará encontrou-se maiores taxas de mortalidade por neoplasias em municípios com predomínio da agricultura industrial irrigada, em relação a municípios com predomínio de agricultura familiar tradicional do semiárido ${ }^{29}$.

A ocorrência de episódio prévio de intoxicação por agrotóxicos é um indicador da exposição a altas doses desses produtos e, neste estudo, foi relatada por parcela não desprezível dos camponeses vivendo sob exposição a agrotóxico no território de agricultura industrial irrigada, sendo significativamente superior à frequência encontrada no grupo de camponeses envolvidos com agroecologia. A chance para episódios prévios de intoxicação por agrotóxicos, foi oito vezes maior entre os camponeses do território da agricultura industrial, em comparação aos camponeses envolvidos com práticas agroecológicas.

Diferentemente da associação bem estabelecida na literatura entre ideação suicida e intoxicação aguda por agrotóxicos, não há evidências epidemiológicas robustas associando depressão/ suicídio com exposição crônica a agrotóxicos, isto é, exposição prolongada e cumulativa a baixas doses destas substâncias ${ }^{26}$. No escopo deste estudo, para tentar responder à pergunta "Intoxicação crônica por agrotóxicos está associada à ideação suicida?", o modelo foi ajustado por outras variáveis que potencialmente poderiam interferir no resultado. Observou-se que ideação suicida, nos últimos 30 dias, manteve-se associada a camponeses vivendo sob exposição prolon- gada e cumulativa a agrotóxicos no território de agricultura industrial irrigada no vale do Gorutuba, independentemente de ter havido episódios pregressos de intoxicação aguda por agrotóxicos, bem como de outros aspectos, como o uso abusivo do álcool, a escolaridade e o estado conjugal, que também se mantiveram distintamente associados às práticas agrícolas dos grupos avaliados. Estes resultados estão em concordância com o estudo transversal, aninhado na coorte Agricultural Health Study nos Estados Unidos, onde foi encontrada associação entre transtornos psiquiátricos e exposição crônica a agrotóxicos ${ }^{33}$.

A plausibilidade biológica da associação entre intoxicação crônica por agrotóxicos e agravos neuropsiquiátricos, foi discutida em estudos de revisão ${ }^{13,18,26}$. Os inseticidas organofosforados, e outras classes toxicológicas de agrotóxicos, sinalizam na direção da plausibilidade dos agrotóxicos estarem na via causal de sintomas depressivos e distúrbios psicoemocionais ${ }^{18}$. $\mathrm{Na}$ intoxicação aguda por estas substâncias, tem-se uma hiperestimulação colinérgica por inibição das enzimas acetilcolinesterases nas sinapses nervosas. Nas intoxicações crônicas, com doses não suficientes para produzir inibição das colinesterases, observaram-se, em estudos experimentais, alterações em outros sistemas neurotransmissores, tais como os sistemas serotoninérgicos, glutamatérgicos, noradrenérgicos e dopaminérgicos ${ }^{26}$. Entre os efeitos da intoxicação crônica estão as alterações do neurodesenvolvimento ${ }^{13}$, depressão, aumento da impulsividade e outras alterações do humor e do comportamento, que poderiam contribuir para ideação suicida e suicídio ${ }^{18}$.

O presente estudo mostrou que camponeses vivendo no território do Projeto de agricultura industrial irrigada, apresentaram maior chance para uso problemático de álcool $(\mathrm{OR}=2,30$; IC95\% 1,18-4,48), em relação a camponeses com práticas agroecológicas, oriundos de comunidades tradicionais do semiárido.

Outros estudos já destacaram que a agricultura industrial moderna, baseada nas monoculturas e no uso intensivo de agrotóxicos, impacta negativamente a saúde mental de camponeses $^{14,19,32,37-39}$, especialmente em países periféricos onde o uso de agrotóxicos é amplamente disseminado e estimulado, mas precariamente controlado e regulamentado ${ }^{1,3,18,36}$.

Os resultados deste estudo devem ser considerados à luz de algumas limitações. A amostragem não foi probabilística por não haver registros oficiais de cadastro populacional para as pequenas comunidades estudadas. Não foram abordados 
alguns fatores de risco conhecidos para ideação suicida/suicídio, tais como antecedentes pessoais e familiares de desordens psiquiátricas e comportamentos suicidas, bem como condições de saúde e eventos estressores não relacionados à agricultura. Adicionalmente, a exposição aos agrotóxicos para as comunidades avaliadas não foi mensurada de forma direta. Apesar dessas limitações, o estudo registra resultados relevantes, que devem subsidiar amplas discussões entre os gestores de saúde e estimular novos estudos para a região.

\section{Conclusão}

Os camponeses vivendo sob condições que ensejam prolongada e cumulativa exposição a agrotóxicos, no território de agricultura industrial irrigada o vale do Gorutuba, tiveram maiores chances para apresentar ideação suicida, nos últi- mos 30 dias, quando comparados a camponeses envolvidos com práticas agroecológicas em comunidades tradicionais do semiárido, independentemente da presença de episódios prévios de intoxicação aguda por agrotóxicos.

Este estudo, ao comparar populações camponesas do território semiárido mineiro, submersas em modelos agrícolas distintos, trouxe à luz a necessidade de se conhecer os determinantes que fazem a agricultura industrial, baseada em monoculturas e uso intensivo de agrotóxicos, estar associada ao sofrimento mental dos camponeses.

As múltiplas conexões entre os campos da saúde e da agroecologia precisam ser mais bemevidenciadas, não somente pela construção e valorização de sistemas alimentares sustentáveis ou pela preservação dos ecossistemas, mas também pelo potencial dessas práticas sustentáveis em produzir, individual e coletivamente, novos sentidos e esperanças no porvir.

\section{Colaboradores}

Todos os autores participaram igualmente da concepção do estudo, análise dos resultados e redação do texto final. CWP Gonzaga foi responsável pela coleta dos dados.

\section{Agradecimentos}

Agradecemos à Coordenação de Aperfeiçoamento de Pessoal de Nível Superior (CAPES) pela concessão de Bolsa de Mestrado. 


\section{Referências}

1. Carneiro FF, Rigotto RM, Augusto LGS, Búrigo AC. Dossiê ABRASCO: um alerta sobre os impactos dos agrotóxicos na saúde. Rio de Janeiro/São Paulo: Expressão Popular; 2015.

2. Porto MF, Soares WL. Modelo de desenvolvimento, agrotóxicos e saúde: um panorama da realidade agrícola brasileira e propostas para uma agenda de pesquisa inovadora. Rev Bras Saude Ocup 2012; 37(125):17-50.

3. Bombardi LM. Geografia do uso de agrotóxicos no Brasil e conexões com a União Europeia. São Paulo: USP; 2017.

4. Bassil KL, Vakil C, Sanborn M, Cole DC, Kaur JS, Kerr KJ. Cancer health effects of pesticides: Systematic review. Can Fam Physician 2007; 53:1704-1711.

5. Curvo HRM, Pignati WA, Pignatti MG. Morbimortalidade por câncer infanto-juvenil associada ao uso agrícola de agrotóxicos no Estado de Mato Grosso, Brasil. Cad Saude Colet 2013; 21(1):10-17.

6. Diamanti-Kandarakis E, Bourguignon JP, Giudice LC, Hauser R, Prins GS, Soto A, Zoeller RT, Gore AC. Endocrine-disrupting chemicals: an Endocrine Society scientific statement. Endocr Rev 2009; 30(4):293-342.

7. Bergman Å, Heindel JJ, Jobling S, Kidd K, Zoeller TR. State of the science of endocrine disrupting chemicals 2012. Genebra: WHO; 2013.

8. Ascherio A, Chen H, Weisskopf MG, O'Reilly E, McCullough ML, Calle EE, Schwarzschild MA, Thun MJ. Pesticide exposure and risk for Parkinson's disease. Ann Neurol 2006; 60(2):197-203.

9. Parrón T, Requena M, Hernández AF, Alarcón R. Association between environmental exposure to pesticides and neurodegenerative diseases. Toxicol ApP Pharm 2011; 256(3):379-385.

10. London L, Beseler C, Bouchard MF, Bellinger DC, Colosio C, Grandjean P, Raul Harari, T Kootbodien, Kromhout H, Little F, Meijster T, Moretto A, Rohlman DS, Stallones L. Neurobehavioral and neurodevelopmental effects of pesticide exposures. Neurotoxicology 2012; 33(4):887-896.

11. Bouchard MF, Bellinger DC, Wright RO, Weisskopf MG. Attention-deficit/hyperactivity disorder and urinary metabolites of organophosphate pesticides. Pediatrics 2010; 125(6):e1270-e1277.

12. Grandjean P, Harari R, Barr DB, Debes F. Pesticide exposure and stunting as independent predictors of neurobehavioral deficits in Ecuadorian school children. Pediatrics 2006; 117(3):e546-e556.

13. Bjørling-Poulsen M, Andersen HR, Grandjean P. Potential developmental neurotoxicity of pesticides used in Europe. Environ Health 2008; 7(1):50.

14. Horrigan L, Lawrence RS, Walker P. How sustainable agriculture can address the environmental and human health harms of industrial agriculture. Environ Health Perspect 2002; 110(5):445-456.

15. Burigo AC, Vaz BA, Londres F, Franco Netto G, Menezes MAC, Pacheco MEL, Souza NA, Petersen P, organizadores. Caderno de estudos: saúde e agroecologia. Rio de Janeiro: Fiocruz, ANA, ABA-Agroecologia; 2019.

16. Altiere MA. Agroecology: the science of natural resource management for poor farmers in marginal environments. Agric Ecosyst Environ 2002; 93(1-3):1-24.
17. Faria NMX, Fassa ACG, Meucci RD. Association between pesticide exposure and suicide rates in Brazil. Neurotoxicology 2014; 45:355-362.

18. London L, Flisher AJ, Wesseling C, Mergler D, Kromhout H. Suicide and exposure to organophosphate insecticides: cause or effect? Am J Ind Med 2005; 47(4):308-321.

19. Meyer A, Koifman S, Koifman RJ, Moreira JC, De Rezende Chrisman J, Abreu-Villaca Y. Mood disorders hospitalizations, suicide attempts, and suicide mortality among agricultural workers and residents in an area with intensive use of pesticides in Brazil. J Toxicol Environ Health A 2010; 73(13-14):866-877.

20. Kim J, Shin DH, Lee WJ. Suicidal ideation and occupational pesticide exposure among male farmers. Environ Res 2014; 128:52-56.

21. Zhang J, Stewart R, Phillips M, Shi Q, Prince M. Pesticide exposure and suicidal ideation in rural communities in Zhejiang province, China. Bull WHO 2009; 87:745-753.

22. Olfson M, Weissman MM, Leon AC, Sheehan DV, Farber L. Suicidal ideation in primary care. J Gen Inten Med 1996; 11(8):447-453.

23. Koh SB, Kim TH, Min S, Lee K, Kang DR, Choi JR. Exposure to pesticide as a risk factor for depression: $\mathrm{A}$ population-based longitudinal study in Korea. Neurotoxicology 2017; 62:181-185.

24. Kim J, Ko Y, Lee WJ. Depressive symptoms and severity of acute occupational pesticide poisoning among male farmers. Occup Environ Med 2013; 70(5):303309.

25. Wesseling C, De Joode BVW, Keifer M, London L, Mergler D, Stallones L. Symptoms of psychological distress and suicidal ideation among banana workers with a history of poisoning by organophosphate or n-methyl carbamate pesticides. Occup Environ Med 2010; 67(11):778-784.

26. Freire C, Koifman S. Pesticides, depression and suicide: a systematic review of the epidemiological evidence. Int J Hyg Environ Health 2013; 216(4):445-460.

27. Ferreira MLPC. A pulverização aérea de agrotóxicos no Brasil: cenário atual e desafios. R Dir Sanit 2014; 15(3):18-45.

28. Bukalasa JS, Brunekreef B, Brouwer M, Vermeulen R, Jongste JC, van Rossem L, et al. Proximity to agricultural fields as proxy for environmental exposure to pesticides among children: The PIAMA birth cohort. Sci Total Environ 2017; 595:515-520.

29. Rigotto RM, Silva AMC, Ferreira MJM, Rosa IF, Aguiar ACP. Tendências de agravos crônicos à saúde associados a agrotóxicos em região de fruticultura no Ceará, Brasil. Rev Bras Epidemiol 2013; 16(3):763-73.

30. Paraná. Secretaria de Estado da Saúde (SES). Protocolo de avaliação das intoxicações crônicas por agrotóxicos [Internet]. 2013 [acessado 2019 mar 23]. Disponível em: http://www.saude.pr.gov.br/arquivos/File/CEST/ Protocolo_AvaliacaoIntoxicacaoAgrotoxicos.pdf.

31. Conigrave KM, Hall WD, Saunders JB. The AUDIT questionnaire: choosing a cut-off score. Addiction 1995; 90(10):1349-1356.

32. Pires DX, Caldas ED, Recena MCP. Uso de agrotóxicos e suicídios no Estado do Mato Grosso do Sul, Brasil. Cad Saude Publica 2005; 21:598-604. 
33. Beseler CL, Stallones L, Hoppin JA, Alavanja MC, Blair A, Keefe T, Kamel F. Depression and pesticide exposures among private pesticide applicators enrolled in the Agricultural Health Study. Environ Health Perspect 2008; 116(12):1713-1719.

34. Pickett W, King WD, Lees RE, Bienefeld M, Morrison HI, Brison RJ. Suicide mortality and pesticide use among Canadian farmers. Am J Ind Med 1998; 34(4):364-372.

35. Pignati WA, Lima FANDS, Lara SSD, Correa MLM, Barbosa JR, Leão LHDC, Pignatti MG. Distribuição espacial do uso de agrotóxicos no Brasil: uma ferramenta para a Vigilância em Saúde. Cien Saude Colet 2017; 22(10):3281-3293.

36. Bull D. A growing problem: pesticides and the Third World poor. Oxford: Oxfam; 1982.

37. Campos Ÿ, Silva VSP, Mello Sarpa MC, Barros UO. Exposure to pesticides and mental disorders in a rural population of Southern Brazil. Neurotoxicology 2016; 56:7-16.

38. Moreira JC, Peres F, Simões AC, Pignati WA, Dores EDC, Vieira SN, Strüssmann C, Mott T. Contaminação de águas superficiais e de chuva por agrotóxicos em uma região do estado do Mato Grosso. Cien Saude Colet 2012; 17(6):1557-1568.

39. Carneiro FF, Pessoa VM, Teixeira ACA. Campo, floresta e águas: práticas e saberes em saúde. Brasília: Ed UnB; 2017.

Artigo apresentado em 07/04/2020

Aprovado em 14/07/2020

Versão final apresentada em 16/07/2020

Editores-chefes: Romeu Gomes, Antônio Augusto Moura da Silva 